\title{
Frontières
}

\section{Marges entre l'école et la vie adulte}

\section{Georgette Goupil, Marc J. Tassé, Émilie Boisseau, Geneviève Bouchard et Catherine Doré}

Volume 14, numéro 1, automne 2001

Où est la marge?

URI : https://id.erudit.org/iderudit/1074149ar

DOI : https://doi.org/10.7202/1074149ar

Aller au sommaire du numéro

Éditeur(s)

Université du Québec à Montréal

ISSN

1180-3479 (imprimé)

1916-0976 (numérique)

Découvrir la revue

Citer cet article

Goupil, G., Tassé, M. J., Boisseau, É., Bouchard, G. \& Doré, C. (2001). Marges

entre l'école et la vie adulte. Frontières, 14(1), 38-42.

https://doi.org/10.7202/1074149ar

\section{Résumé de l'article}

Toute personne, au cours de son existence, vivra des transitions en traversant les différentes étapes de sa vie. Cet article présente l'une de ces transitions, soit la transition entre l'école et la vie adulte. La transcription de réunions de plans de transition d'élèves ayant une déficience intellectuelle a permis de décrire les rêves, les craintes et les dilemmes auxquels les jeunes et leur famille font face lorsque ces élèves quittent l'école. L'étude met aussi en évidence la nécessité d'offrir une planification à long terme qui permette une transition harmonieuse entre les deux milieux de vie. 


\section{RÉSUMÉ}

Toute personne, au cours de son existence, vivra des transitions en traversant les différentes étapes de sa vie. Cet article présente l'une de ces transitions, soit la transition entre l'école et la vie adulte. La transcription de réunions de plans de transition d'élèves ayant une déficience intellectuelle a permis de décrire les rêves, les craintes et les dilemmes auxquels les jeunes et leur famille font face lorsque ces élèves quittent l'école. L'étude met aussi en évidence la nécessité d'offrir une planification à long terme qui permette une transition harmonieuse entre les deux milieux de vie.

Mots clés : autisme - deuil-plans de transition

\section{ABSTRACT \\ Everyone, during his or her lifetime, will experience transitions while going through various life stages. This article presents one of these transitions, namely the transition between school life and adult life. Taking down the minutes for the transition plan meetings for mentally retarded students has enabled us to describe dreams, fears and dilemmas that young adults and their families must face when such students leave school. This study also highlights the need to offer long-term planning that permits a harmo- nious transition between the two milieus.}

Key words : autism - grief - transition plans

\section{MARGES ENTRE L'ÉCOLE ET LA VIE ADULTE}

Georgette Goupil, Ph.D., professeure, Département de psychologie, UQAM.

Marc J. Tassé, Ph.D., Center for Development and Learning, University of North Carolina.

\section{Émilie Boisseau, Geneviève Bouchard et Catherine Doré, \\ Département de psychologie, UQAM.}

Les familles d'enfants qui sont handicapés passent par différentes étapes ou cycles de vie.

Plusieurs de ces transitions sont marquées par le stress des décisions à prendre et des nouvelles adaptations au sein de la famille. Selon Bouchard et al. (1994), ce processus débute dès que le diagnostic est posé. Selon ces auteurs, la famille devra s'adapter, apprendre à vivre avec la différence et ses exigences. Pour plusieurs parents, le diagnostic a lieu au moment de la naissance. Ginette Beaulieu décrit ainsi cet instant chargé d'émotion pour la famille :

Un enfant va naître. L'enfant imaginé, désiré, tant attendu. Fruit d'un désir, du rêve d'un couple qui envisage de vivre heureux avec un enfant en santé, beau [...] mais soudain... la terre bascule! La fête de la naissance prend l'allure d'une tragédie. L'enfant tant désiré est malformé, handicapé à vie. Accident de la nature ou risque prévisible... c'est de toute façon la consternation, l'effondrement du rêve. Le choc émotif qui s'ensuit est particulièrement douloureux et pénible, quasi insoutenable. Les parents font face à cette situation irréversible, irrémédiable, comme on vit un échec ou un deuil.

(Beaulieu, 1983, p. 1).
Pelchat (1992) dépeint également ce moment en parlant de l'effondrement d'un grand rêve. Pour cette auteure, plus l'enfant réel est différent des rêves des parents, plus l'adaptation sera difficile. Pelchat (1990) souligne que les parents s'adapteront au travers d'un cheminement, d'un processus de deuil de l'enfant désiré. C'est ainsi qu'ils s'ajusteront à l'enfant réel. Cette auteure souligne que le processus de deuil de l'enfant parfait est nécessaire: «Ceux-ci doivent donc faire le deuil de cet enfant désiré et imaginé parfait. Plus grand est l'écart entre les fantasmes et les aspirations visant l'enfant pendant la grossesse et la réalité après l'accouchement, plus le processus de deuil est profond et difficile » (Pelchat, 1990, p. 45-46).

Turnbull et Turnbull (1990) indiquent eux aussi que l'enfant parfait est décédé et qu'à sa place, il y a un bébé qui présente des problèmes qui créent de l'anxiété pour les parents. De plus, ces derniers ignorent souvent les perspectives d'avenir de leur enfant, plusieurs n'osent plus se projeter dans le futur ne sachant ce que leur enfant pourra réaliser comme travail ou comme études avec sa déficience. Le cheminement à travers le processus de deuil permettra aux parents, selon Bouchard et al. (1994), de mieux identifier leurs émotions, leurs ressources et de chercher à l'extérieur l'aide et le soutien nécessaires. Pelchat décrit ainsi l'importance pour les parents de franchir cette étape: «le processus de deuil et la résolution de la perte représentent une étape essentielle à l'adaptation puisqu'ils aident les parents à s'attacher à l'enfant réel et à en prendre soin » (cité dans Bouchard et al., 1994, p. 20). Le processus peut être entravé, compliqué par la nécessité de répondre à des besoins importants de l'enfant : les soins sont souvent plus complexes, il y 
a nécessité de recourir à divers services et programmes d'intervention précoce.

À partir de la naissance de l'enfant, la famille devra donc s'engager dans différentes étapes. En général, selon Bouchard et al. (1994), la famille traversera les stades émotifs suivants : choc, négation, détresse, réorganisation, adaptation à la différence et acceptation de l'enfant. La naissance ou l'annonce du diagnostic n'est pas la seule période difficile pour les familles. Les passages d'une étape de vie à une autre (de l'enfance à l'adolescence par exemple) obligeront la famille à s'adapter aux changements qui seront, pour plusieurs, de nouvelles sources de stress. Chacune de ces périodes créera des défis et souvent amènera les parents à faire de nouveaux deuils. Même si les politiques en matière d'adaptation scolaire prônent l'intégration en classe ordinaire, plusieurs parents auront à inscrire leur enfant dans des mesures spéciales de scolarisation renonçant ainsi à une scolarisation en classe ordinaire. Plusieurs enfants seront alors scolarisés à l'extérieur de leur quartier, dans des écoles ou des classes spéciales. Ici encore, les parents devront renoncer à voir l'enfant tisser les mêmes liens d'amitié à l'école et dans son quartier.

L'adolescence, le passage à la vie adulte créent de nouvelles situations générant de nouvelles craintes pour les parents : craintes liées à la vulnérabilité de leur enfant, problèmes liés à l'acceptation par la communauté, etc. Plusieurs parents auront dû aussi faire d'autres deuils. En effet, plusieurs renonceront à voir leur enfant compléter un cours professionnel, des études collégiales ou universitaires; d'autres renonceront à voir vivre leur enfant de manière autonome en appartement, etc. Le stress créé par les transitions entre les périodes de vie suscite de plus en plus d'intérêt dans la littérature, entre autres, parce que plusieurs cherchent des moyens d'intervention pour rendre ces périodes plus faciles pour les familles et leurs enfants qui ont une déficience.

Bien sûr, toute personne, au cours de son existence, vivra des transitions en traversant les différentes étapes de sa vie. Blalock et Patton (1996) parlent de transitions verticales pour exprimer les changements entre une période de vie et une autre, ou encore de transitions horizontales pour désigner des changements qui ont lieu à l'intérieur d'une même période, tel le passage d'une école spéciale à une école ordinaire. Cet article traite plus particulièrement de la transition entre l'école et la vie adulte. Cette transition représente un passage important pour tout élève. Mais dans le cas de jeunes adultes ayant un retard intellectuel, l'insertion sociale et professionnelle se vit parfois difficilement. En effet, que ce soit dans la sphère du travail, du logement ou des loisirs, ces jeunes adultes doivent composer avec de nombreux services rattachés à différents établissements et la coordination ne se fait pas toujours sans heurt. De plus, l'appropriation de ces différentes sphères de la vie nécessite de nouveaux apprentissages.

\section{DÉFINITION DE LA TRANSITION DE L'ÉCOLE À LA VIE ADULTE}

Selon Wehman (1996), la transition entre l'école et la vie adulte se définit comme des changements, des ajustements et des expériences dans la vie des jeunes adultes. Alors que plusieurs des jeunes ayant un retard intellectuel aimeraient eux aussi une vie sociale enrichissante et une insertion au monde du travail, le portrait que dépeint la réalité est parfois tout autre. Souvent, après la dernière année de scolarisation, ces jeunes se retrouvent coupés de plusieurs services offerts à l'école et des liens créés avec leurs amis et les différents intervenants de l'école. Aussi, selon Affleck et al. (1990), certains élèves n'ont pas les habiletés nécessaires afin de trouver ou de garder un emploi stable et même ceux qui y parviennent n'ont souvent pas un salaire assez élevé pour subvenir à leurs besoins. Au Québec, dans une étude auprès de 26 jeunes ayant une déficience intellectuelle et ayant terminé leurs études dans les années précédentes, Vendittoli et Gamache (1997) précisent qu'en général, ces jeunes n'ont pour seul débouché que les milieux de travail protégés et qu'une infime minorité conservent un emploi ordinaire une fois terminées les subventions spéciales accordées aux entreprises pour les embaucher. Ces auteurs constatent que ces jeunes consacrent 22,4 heures par semaine au travail, 19,9 heures à la vie domestique et 43,2 heures aux loisirs. Ces auteurs rapportent également que le style de vie varie selon le type de résidence du jeune. La transition sera favorisée lorsque le jeune développe différentes facettes de sa vie d'adulte: loisirs, travail, relations sociales, etc.

Cette transition est aussi en lien avec l'autodétermination, cette capacité d'une personne à agir volontairement selon ses décisions. Être autodéterminé, c'est être en mesure de se fixer des objectifs et être motivé à les atteindre. Wehmeyer (1995) établit quatre caractéristiques d'une action autodéterminée: 1) l'individu agit de manière autonome; 2) ses comportements sont autorégulés; 3 ) la personne crée des

\section{PARLONS UN PEU DE... LA COMMUNICATION}

"Je suis une jeune adolescente de seize ans [...] Je parlerai ici plus particulièrement de l'abandon des adolescents par leurs parents, de la culpabilité des parents et des parents trop envahissants. [...]

Les parents doivent toujours demeurer en bons termes avec leurs enfants. C'est pour cela que je dis que certains parents ont raison de se sentir coupables lorsque leurs jeunes glissent sur la pente de la délinquance, car c'est souvent de leur faute. Deuxièmement, j'aimerais vous parler des parents qui n'ont pas à se sentir coupables quand ils ne le devraient pas. Ça peut paraître contradictoire, mais un adolescent n'aura ni respect ni appréciation pour des parents qui se culpabilisent à propos de rien, puisque ceci démontre une certaine fragilité de caractère. Par contre, certains parents posent des gestes fatals et sont responsables même s'ils n'ont pas voulu mal faire. [...] Troisièmement, je vais vous parler des parents trop envahissants qui, eux aussi, devraient se responsabiliser par rapport à leur attitude. Ce qui peut déplaire excessivement à l'adolescent, c'est de se sentir surveillé de près ou espionné par des parents envahissants. [...]

En conclusion, je pense sincèrement que les parents qui ne se culpabilisent pas quand ils posent des gestes désagréables envers leurs enfants ont tort. Ils devraient assumer une grande partie des responsabilités. D'ailleurs, le parent ne devrait-il pas se réserver un peu plus de temps pour être en compagnie de son enfant?»

Marisol Hamel

"Je l'avoue, il est vrai que les jeunes s'enferment souvent dans leur chambre lorsque vient le temps communiquer, mais ce serait une énorme erreur que de les laisser se retirer. [...] Peut-être que les parents élèvent trop la voix ou bien qu'ils sont parfois blessants dans leurs remarques? En résumé, il est important de ne jamais abandonner la communication avec les adolescents, même s'ils ne sont pas toujours consentants. Se retirer dans leur chambre représente tout simplement du réconfort, de l'isolement, et non une tactique de rupture. [...] La prose d'opposition des jeunes est déjà un bon début à la communication. Bref, les parents ne doivent pas toujours donner raison aux ados, mais ils doivent absolument les écouter en tout temps. »

Sarah Vaillancourt 
événements et y répond de manière personnelle; 4) ses actions contribuent à son épanouissement.

L'autodétermination est liée de près aux mouvements de réinsertion ou d'intégration sociale des personnes handicapées (Lachapelle et Boisvert, 1999). Pour Wehmeyer et Schwartz (1998), la promotion de l'autodétermination passe par une éducation qui permet à l'individu d'acquérir plus de contrôle sur sa vie, de faire des choix et de prendre des décisions basées sur ses goûts, de se donner des buts d'accomplissement personnel et de faire des apprentissages pour résoudre des problèmes et assumer davantage de responsabilités. Ces auteurs insistent également sur le fait que les adultes autodéterminés ont une meilleure qualité de vie.

Wehman (1996) indique que la planification de la transition doit être d'abord centrée sur la personne, ce qui implique que l'on doive considérer ses besoins, ses choix, mais aussi l'importance des systèmes d'aide formels et informels. Dans un tel contexte, toujours selon Wehman, ce sont les activités quotidiennes, de même que les liens entre sa famille et sa communauté, qui deviennent importants. C'est donc l'action d'un réseau entier qui est requise, car une seule personne ne peut assumer à elle seule tout ce qu'exige la transition. En effet, comme l'indique justement O'Leary (1998), l'école ne peut pas tout faire.

$\mathrm{Au}$ cours des dernières années, notre équipe de recherche s'est particulièrement intéressée à la transition entre l'école et la vie adulte de jeunes adultes ayant une déficience intellectuelle. Puisque ces jeunes sont plus à risque de se retrouver en marge du système dès la fin de leurs études, il est impératif de se demander comment ce passage vers la vie adulte pourrait être facilité. Nous nous sommes donc penchés sur cette question à travers l'implantation de plans de transition, c'est-à-dire une planification à long terme du passage entre l'école et la vie adulte.

\section{LE PLAN DE TRANSITION}

La transition entre l'école et la vie adulte n'est pas un sujet nouveau. Dès le début des années 1980, les Américains développent des modèles qui, à cette époque, sont surtout centrés sur l'intégration au marché du travail. En 1988, Brotherson et al. observent que les parents sont surtout préoccupés par les soins de base accordés à leur enfant, à son lieu de résidence, ses loisirs et ses habiletés de socialisation. Le travail, bien qu'il soit aussi une préoccupation, n'est pas la première. Les modèles ont donc par la suite évolué en incluant non seulement le travail mais aussi différentes dimensions de la vie : le logement, l'insertion

À L'ÂGE OÙ LA MAJORITÉ DES PARENTS

APPRENNENT À SE DÉTACHER DE LEUR ENFANT

AFIN DE LUI CONFÉRER PLUS D’INDÉPENDANCE,

LES PARENTS D'ENFANTS AYANT UNE DÉFICIENCE INTELLECTUELLE

DOIVENT CONTINUER À S'IMPLIQUER.

dans la communauté, les loisirs, etc. Aux États-Unis, le plan de transition fait partie intégrante de la législation sur la planification des interventions pour les élèves en difficulté (IDEA - PL 105-17, 1997). L'école, en collaboration avec la famille, identifie les objectifs et les moyens d'intervention qui faciliteront le passage du jeune de l'école à la vie adulte et le plan de transition doit débuter au moins cinq ans avant que l'élève ne quitte l'école.

Selon la définition américaine, le plan de transition est un ensemble d'activités planifiées à l'intention de l'élève. Il s'agit d'un processus orienté pour faciliter le passage de l'école aux activités postsecondaires. Ces activités incluent: l'éducation postsecondaire, l'initiation au travail, l'intégration à un emploi, l'utilisation des services d'éducation aux adultes, l'autonomie personnelle et la participation aux activités communautaires.

Si les Américains ont prévu une telle mesure dans leur législation (IDEA, 1997), au Québec il n'y a pas, dans la Loi sur l'instruction publique (1990) de mention concernant la transition. Ce concept étant relativement nouveau en milieu francophone, en 1997 nous entreprenions une étude auprès de 21 élèves ${ }^{1}$ présentant un retard mental et tous scolarisés dans des écoles ou des classes spéciales. Dans le cadre de ce projet, nous avons offert divers outils aux écoles et aux parents pour élaborer les plans de transition (Forest et Pearpoint, 1991; Hobbs et Allen, 1989; Luckasson et al., 1992). Un outil particulièrement apprécié des milieux fut le Mapp de Forest et Pierpoint. Le Mapp propose une démarche systématique où l'on retrace d'abord l'histoire du jeune. Par la suite, les participants et le jeune s'expriment sur les rêves, les craintes, les besoins du jeune et déterminent par ordre de priorité les interventions subséquentes.

Les plans de transition ont été réalisés au cours de réunions regroupant les parents, le personnel concerné de l'école, l'élève (présent dans $53 \%$ des réunions) et parfois des intervenants d'autres organismes (ex. : centre de réadaptation). Nous avons enregistré les réunions de plans de transition de ces jeunes adultes. Nous vous livrons ici quelques témoignages, certes partiels, mais qui attestent de l'existence de cette marge entre l'école et la vie adulte. Le contenu de ces réunions de transition nous a aussi amenés à observer plusieurs des constats faits dans la littérature à propos des rêves, inquiétudes et dilemmes, souvent difficiles à résoudre, de ces jeunes adultes et de leur famille.

\section{RÊVES ET CRAINTES}

$\mathrm{Au}$ cours des réunions, les parents et les élèves s'expriment sur leurs rêves et leurs craintes. Lorsque les parents de ces jeunes ayant une déficience intellectuelle pensent à l'avenir de leur enfant, leurs espoirs ne sont pas différents de ceux de tous les parents. Ils ont bien sûr le rêve que leur enfant soit assez autonome pour pouvoir un jour voler de ses propres ailes, s'épanouir, trouver un sens à sa vie et se sentir utile et aimé. Voici quelques témoignages à ce sujet: "Je veux qu'il continue à aller à l'école, mais qu'il puisse évoluer aussi. » «Je veux qu'elle parvienne à être sur le marché $\mathrm{du}$ travail et qu'elle soit autonome financièrement pour pouvoir négocier avec les problèmes pouvant survenir. » Ces rêves ne sont par contre pas exempts de doutes: "C'est sûr que tu as toujours peur qu'elle ne travaille pas, qu'elle ne puisse rien faire, qu'elle reste à la maison. »

Dans les réunions de plans de transition, il est fréquent que l'on demande aux parents le travail souhaité pour leur enfant. Plusieurs répondent tout simplement «un travail comme tout le monde». Un parent dira : "Je ne pense pas qu'il y ait un endroit qu'il aimerait spécifiquement, mais nous, on aimerait qu'il continue à aller à l'école ou qu'il trouve un emploi pour donner un sens à sa vie. Il ne faut pas que ça s'arrête là. C'est sûr qu'il peut rester à la maison tout seul. Mais nous, on calcule que ce n'est pas une vie. Une personne a besoin de se sentir utile. »

Quant aux élèves, ils ont eux aussi des rêves semblables à tous les autres jeunes. Ils veulent devenir épicier, infirmière, vétérinaire, concierge, cuisinier, éducatrice, jardinier, etc. Certains ont le désir de vivre en couple, de fonder une famille, d'avoir leur chez-soi et de conduire leur automobile.

Parallèlement, de nombreuses craintes surgissent lors de la transition. Thorin et 


\section{PARLONS UN PEU DE... L'AUTORITÉ PARENTALE}

"L'autorité parentale est souvent mal vue de la part du jeune. Même s'il n'a pas atteint sa majorité, ses parents n'ont pas tous les droits sur lui. Les sorties, les fréquentations et le choix de carrière sont souvent des sujets qui amènent une chicane.

Certes il faut des restrictions par rapport aux sorties, mais sans exagération. Les adolescents sont souvent imprévisibles, ils commencent à vouloir voler de leurs propres ailes. Les adolescents ne sont-ils pas assez mûrs pour s'amuser? Personnellement, je crois que même les enfants de cinq ans ont la maturité de faire la fête. Sauf, qu'il y a une différence entre s'amuser à l'âge de trois ou quatre ans et à l'âge de quinze ou seize ans. [...]

Plusieurs adultes se demandent comment mieux connaître les amies et amis de leurs enfants. Une réponse bien simple: ne pas les juger au premier regard et discuter avec eux comme s'ils étaient des amis. Souvenez-vous que l'apparence est souvent trompeuse, ne soyez pas trop sévères lorsqu'il s'agit des fréquentations.

Il y a également des personnes qui sont dans une situation où les parents veulent décider de leurs projets d'avenir. [...] Le choix de carrière peut facilement être un point de désaccord entre les parents et les adolescents, et pourtant, ça ne devrait pas être ainsi.

Pour clore sur le sujet, si vous avez un enfant, ne faites pas comme s'il était votre possession absolue. Oui, vous avez des droits sur lui, mais il a droit à sa liberté comme tous les autres humains. N'oublions pas que cette jeune personne deviendra adulte sous peu. Elle est en âge de décider ce qui est bien pour elle et ce qui ne l'est pas. Mon seul souhait serait que vous, les parents, laissiez plus de liberté à vos enfants. Il est peut-être temps pour eux de voler de leurs propres ailes. »

Cindy Jackson

"En premier lieu, je crois que c'est à l'adolescence que les jeunes ont besoin d'être encadrés et dirigés par leurs parents. [...] Pour certains, l'adolescence est un véritable enfer, un univers terne et sans couleur où ils ont l'impression de couler et de s'enfoncer. Les parents peuvent alors servir de bouée. [...] Voyez-vous, si l'adolescent commence graduellement à prendre ses responsabilités, il se dira qu'après tout, ce n'est pas un si lourd fardeau. Tandis que s'il se retrouve avec des paquets de responsabilités sur les bras, ce sera trop lourd à porter et les paquets commenceront à tomber. Puis, de fil en aiguille, il se retrouvera les mains vides, convaincu que tout cela n'en vaut pas la peine et il abandonnera. Donc, même s'il faut que les jeunes se responsabilisent, il ne faut pas leur en mettre trop sur le dos. Ils ne sont pas totalement prêts à cela.»

Marie-Andrée Létourneau

"Tout d'abord, la fonction parentale est de servir de refuge, de contenir, de limiter et de permettre les expériences. Je vous concède que les adolescents ont besoin d'autorité, mais je ne vois pas dans cette définition quoi que ce soit qui dit qu'il faudrait tout décider à leur place. Au contraire! Il faut permettre les expériences. Ce n'est pas en les contrôlant qu'ils vont s'épanouir et devenir plus indépendants. C'est alors pourquoi je crois qu'il faut trouver un juste milieu à l'utilisation de l'autorité; ne pas trop être permissif, mais ne pas abuser de ce pouvoir non plus.» Émilie Champigny-A

Irvin (1992) indiquent que la transition soulève beaucoup d'inquiétude et de stress dans les familles. Lors des réunions de plans de transition, nous avons constaté que certains parents redoutent le moment où ils ne seront plus là pour veiller sur leur enfant.

"Qu'arrivera-t-il lorsque nous ne serons plus là? Qui va s'occuper de notre enfant? Même s'il reste dans son milieu familial, c'est sûr qu'il va falloir penser un jour à prévoir quelqu'un pour s'occuper de lui. »

«Même s'il reste dans notre famille, ce ne sera pas facile de trouver quelqu'un qui va le prendre en charge. Ce n'est pas évident. Il y a sa sœur qui nous a toujours
- J'ai peur. Sera-t-elle capable de subvenir à ses besoins? »

- Il ne faut pas qu'elle se fasse exploiter, que les employeurs profitent d'elle.»

- Je souhaite qu'il soit capable de se débrouiller seul et qu'il parvienne à prendre contact avec des personnesressources. Je voudrais qu'il soit capable de demander de l'aide s'il est mal pris. C'est important qu'il sache quoi faire. »

Malgré les efforts des organismes venant en aide aux personnes handicapées, plusieurs parents s'inquiètent encore de l'acceptation de leur enfant par la société. Ainsi, un parent dira: "Est-ce que les gens de notre municipalité sont ouverts à engager un jeune comme lui?»

\section{DILEMMES DES PARENTS}

Devant les obstacles qui se dressent face à l'intégration professionnelle et sociale de leur enfant et devant les nombreux apprentissages qui doivent encore être faits pour que le jeune acquière l'autonomie nécessaire à l'atteinte de ses rêves, le parent se retrouve bien souvent face à de cruels dilemmes. Il doit s'impliquer, souvent plus qu'un parent qui n'a pas d'enfant handicapé, afin de l'encadrer dans cette transition. À l'âge où la majorité des parents apprennent à se détacher de leur enfant afin de lui conférer plus d'indépendance, les parents d'enfants ayant une déficience intellectuelle doivent continuer à s'impliquer.

Dans ce contexte, Thorin et al. (1996) soulignent des dilemmes auxquels font face les familles. Voici quelques-uns des dilemmes mentionnés par ces auteurs associés à certains témoignages de parents tirés des plans de transition de notre étude.

\section{- Vouloir offrir au jeune adulte des} occasions d'acquisition

d'indépendance et s'assurer en même temps que ses besoins en matière de santé et sécurité sont satisfaits.

Il peut être nécessaire pour se rendre à son travail ou à une activité de loisir que le jeune prenne le transport en commun seul. Mais certains parents s'inquiètent de la sécurité de leur enfant lors de ces déplacements : "On a de la difficulté à le laisser aller face au danger. Par exemple, il ne regarde pas toujours avant de traverser les rues. On n'est pas convaincu qu'il va réussir à prendre conscience du danger. »

\section{- Vouloir vivre sa propre vie} comme parent tout en s'assurant que le jeune bénéficie d'une certaine qualité de vie.

La présence d'un jeune adulte handicapé demande beaucoup d'attention et de temps de la part de la famille, ce qui crée parfois des situations difficiles pour les parents, 
c'est-à-dire de choisir entre répondre aux besoins du jeune ou à leurs propres besoins. Une mère explique que son principal rôle est celui d'être une mère et que sa vie est planifiée en fonction des besoins de son enfant. Elle dit ne vivre et travailler que pour son enfant. Elle souhaiterait avoir du temps pour elle, pour ses activités tout en répondant aux besoins de sa fille. Une autre mère verbalise ainsi son abattement: «C'est toujours à recommencer, c'est décourageant! Ça fait peut-être deux ans que j'ai arrêté de me battre. Je ne veux pas dire que ce n'est pas ma priorité, mais j'ai un emploi à temps plein. J'ai aussi ma santé. Il faut vivre! »

\section{- Vouloir assurer une vie stable} au sein de la cellule familiale tout en faisant face aux besoins sans cesse changeants

\section{du jeune adulte et de sa famille.}

Plusieurs parents aimeraient que leur vie soit simple, cependant, elle ressemble plutôt, selon quelques-uns, à une course à obstacles. Ces jeunes adultes ont des besoins changeants et parfois exigeants, les parents doivent s'y adapter. Il faut constamment trouver les bons services, penser à prendre les rendez-vous, aller aux rencontres de l'école, etc. Les horaires des parents sont donc déjà chargés et, en plus, il faut s'ajuster aux changements de dernière minute. La transition requiert beaucoup de temps et de planification si la famille veut joindre les deux bouts.

- Vouloir créer une vie sociale pour le jeune adulte tout en essayant de diminuer les interventions dans la vie de ce dernier.

La plupart des parents souhaitent que leur enfant ait un réseau d'amis pour partager certaines activités (sport, cinéma, etc.) et pour contrer la solitude. Un tel réseau leur semble un facteur de protection. Les parents doivent alors leur proposer des occasions pour faire des rencontres ou des activités, tout en souhaitant diminuer la supervision qu'ils exercent auprès de leur enfant.

\section{- Vouloir éviter l'épuisement parental tout en s'assurant du bien-être du jeune adulte.}

Prendre le temps de montrer au jeune adulte comment se débrouiller à la maison et dans la communauté demande des efforts et du temps. De plus, les parents sont parfois épuisés à la fin de leur journée de travail. Certains disent préférer alors faire les choses à la place du jeune au lieu d'attendre qu'il arrive à le faire lui-même. Si l'autonomie dans les apprentissages n'a pas été acquise en collaboration avec l'école avant la fin des études, la transition à la vie adulte risque d'être plus difficile et pour le jeune et pour ses parents.

La description de ces quelques dilemmes illustre les défis auxquels font face les jeunes et leur famille dans ce processus. L'analyse des propos tenus par les jeunes et leur famille lors de ces réunions indique la nécessité d'implanter des démarches plus structurées de transition, de les personnaliser et de les insérer dans le contexte de la communauté. La marge entre l'école et la vie adulte est là et l'arrimage entre les deux contextes de vie s'avère essentiel. Comme l'ont démontré notre étude ainsi que l'expérience américaine depuis déjà une vingtaine d'années, le plan de transition devrait faire partie de la démarche pour tout élève présentant une déficience intellectuelle ou une autre difficulté.

\section{Bibliographie}

AFFLECK, J.Q., EDGAR, E., LEVINE, P. et KORTERING, L. (1990). "Postschool status of students classified as mildly mentally retarded, learning disabled, or nonhandicapped: Does it get better with time?", Education and Training in Mental Retardation, p. 315-324.

BEAULIEU, G. (1983). Le rêve éclaté. Un avis du Conseil des affaires sociales et de la famille sur l'accueil à l'enfant atteint de déficience, Québec, Direction générale des publications gouvernementales du ministère des Communications.

BLALOCK, G. et PATTON, J.R. (1996). «Transition and students with learning disabilities: Creating sound futures ", Journal of Learning Disabilities, vol. 29, n 1, p. 7-16.

BOUCHARD, J.M., PELCHAT, D., BOUDREAULT, P. et LALONDE-GRATON, M. (1994). Processus d'adaptation et qualité de vie de la famille, Montréal, Guérin universitaire.

BROTHERSON, M.J., TURNBULL, A.P., BRONICKI, G. J., HOUGHTON, J. GORDON, C.R., SUMMERS, J.A. et TURNBULL, H.R. (1988). "Transition into adulthood: Parental planning for sons and daughters with disabilities ", Education and Training in Mental Retardation, vol. 23, n 3, p. 165-174.

FIELD, S. (1996). «A historical perspective on student involvement in the transition process: Toward a vision of self-determination for all students », CDEI, vol. 19, n 2, p. 169-176.

FOREST, M. et PEARPOINT, J. (1991). The MAPs Process: An Information Gathering / Planning Tool, Toronto, Inclusion Press.

GOUPIL, G., et al. (2000). "Démarches de plans de transition: de l'école à la vie adulte», Revue francophone de la déficience intellectuelle. Actes Colloque Recherche Défi IQDM, Numéro spécial, vol. 11, p. 50-54.

GOUVERNEMENT DU QUÉBEC (1990). Loi sur l'instruction publique, Québec, Éditeur officiel du Québec.

HOBBS, T. et ALLEN, W.T. (1989). Preparing for the Future: A Practical Guide for Developing Individual Transition Plans, Rohnert Park, CA, California Institute on Human Services, Sonoma State University.

IDEA (1997). Individuals with Disabilities Education Act Amendments of 1997, P.L. 105-17, 20 U.S.C. 1400 et seq.
LACHAPELLE, Y. et BOISVERT, D. (1999). "Développer l'autodétermination des adolescents présentant des difficultés d'apprentissage ou une déficience intellectuelle en milieu scolaire», Revue canadienne de psycho-éducation, vol. 28, nº 2, p. 163-169.

LUCKASSON, R, COULTER, D.L., POLLOWAY, E.A., REISS, S., SCHALOCK, R.L., SNELL, M.E., SPITALNIK, D.M. et STARK, J.A. (1992). Mental Retardation: Definition, Classification, and Systems of Supports (9th Ed.), Washington, D.C., American Association on Mental Retardation.

O'LEARY, E. (1998). Transition: Terms and Concepts, Des Moines, IA, Drake University, ED 419330.

PELCHAT, D. (1992). «Processus d'adaptation des parents d'un enfant atteint d'une déficience et élaboration d'un programme d'intervention précoce à leur intention", Revue canadienne de santé mentale communautaire, vol. 11, no 1, p. 63-80.

PELCHAT, D. (1990). «Problématique familiale lors de la naissance d'un enfant atteint d'une déficience physique ", Revue canadienne de santé mentale communautaire, vol. 9,2, p. 45-59.

THORIN, E.J., YOVANOFF, P. et IRVIN, L. (1996). «Dilemmas faced by families during their young adults' transitions to adulthood: A brief report», Mental Retardation, vol. 34, n 2, p. 117-120.

THORIN, E.J. et IRVIN, L.K. (1992). «Family stress associated with transition to adulthood of young people with severe disabilities », $J A S H$, vol. $17, \mathrm{n}^{\circ} 1$, p. 31-39.

TURNBULL, A.P. et TURNBULL, H.R. (1990). Families, Professionals, and Exceptionality: A Special Partnership, Toronto, Maxwell Macmillan Canada Inc.

VENDITTOLI, M. et GAMACHE, M. (1997). Relance chez les anciens élèves ayant une déficience intellectuelle, Rapport à l'intention du ministère de l'Éducation du Québec et des commissions scolaires de l'Île.

WEHMAN, P. (1996). Life Beyond the Classroom. Transition Strategies for Young People with Disabilities, Baltimore, MA, Paul H. Brookes Publisching Co.

WEHMEYER, M.L. (1995). The Arc's SelfDetermination Scale. Procedural Guidelines, Arlington, TE, The Arc of the United States.

WEHMEYER, M.L. et SCHWARTZ, M. (1998). "The self-determination focus of transition goals for students with mental retardation », $C D E I$, vol. $21, \mathrm{n}^{\circ} 1$, p. 75-86.

\section{Notes}

1. Des questionnaires structurés ont été administrés aux participants de cette étude. Ces données ont été présentées dans un article soumis au British Journal of Special Education et ont fait l'objet d'une présentation à l'AQIS (GOUPIL, G. et al., 2000, p. 50-54). 